\title{
Hongos del Caribe nicaragiiense. Resumen de especies de dos estudios hechos en la Reserva Biológica Indio Maizi
}

\author{
Iris Lilliam Saldívar Gómez \\ Universidad Americana (UAM)
}

\begin{abstract}
Antecedentes
Se estima que los hongos representan aproximadamente 1.5 millones de especies en el mundo (Hawksworth y Rossman 1997), sin embargo, en la actualidad sólo han sido identificadas cerca de cien mil. Ello significa únicamente el $7 \%$, de las especies de hongos a escala global (Piepenbring 2012). Los hongos son un grupo poco investigado en el trópico. Hasta el momento se conoce por debajo del $4 \%$ de las especies que los ecosistemas de esta región podrían albergar (Piepenbring y Ruiz 2008). La riqueza de especies de hongos documentada para el trópico sigue siendo inferior a la de muchos países en el hemisferio norte. Esto está posiblemente ligado a que se han realizado menos esfuerzos muestreales y estudios detallados (Piepenbring 2012).

Los hongos son organismos heterótrofos, lo cual significa que necesitan de un sustrato que los provea de alimento ya que, a diferencia de las plantas, ellos no pueden transformar la energía en azúcares a través de la fotosíntesis. El reino fungi tiene una invaluable importancia en los bosques, debido a su potencial simbiótico con las plantas vasculares y su función de descomponedores de materia orgánica, principalmente madera en descomposición (Egli 2011; Piepenbring 2012). Las funciones de los hongos en la naturaleza son muy complejas, se sabe que aproximadamente un tercio de los hongos conocidos son parásitos (Piepenbring 2012). Unas tres mil especies de hongos son descritas como comestibles y unas sesenta utilizadas en la industria con diferentes aplicaciones (Bolaños y Soto 2011).
\end{abstract}

\section{Los hongos en las culturas precolombinas}

En las culturas precolombinas, tales como la olmeca, azteca, zapoteca y maya, utilizaron hongos no solo en su dieta alimenticia, sino
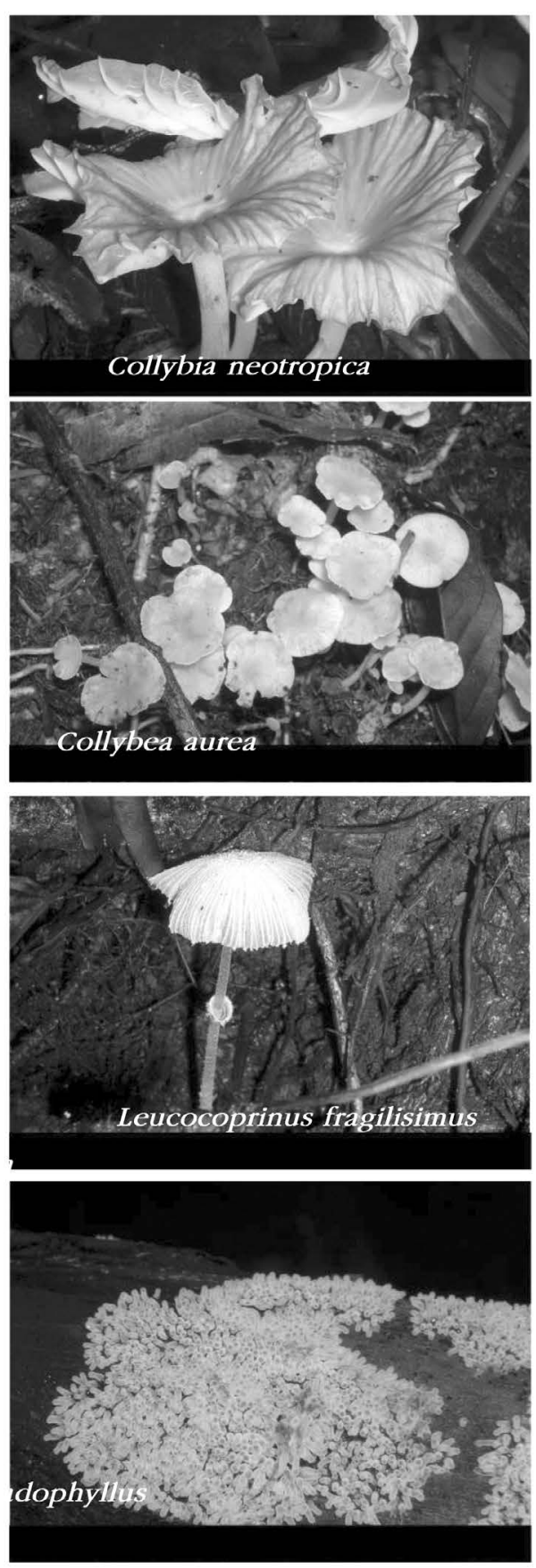
que también tenían importantes usos en diferentes tipos de rituales y ceremonias. El género Psylocibe fue quizás el más usado, ya que comprende 54 especies que crecen en México. Así, su uso en rituales quedó grabado en diferentes petroglifos de coronaciones de caciques aztecas. Los mayas llamaban al Psylocibe cubensis, el teonanacatl y los aztecas lo denominaban k'aizalaj Okox. En rituales también era popular utilizar otras especies como Paneollus y Stropharias (Carod 2015).

Se ha encontrado registro antropológico del uso de hongos en rituales en México, Guatemala, Honduras y el Salvador. La marcada influencia cultural que aun existe de mayas en los dos primeros países, hacen que la recolección de hongos sea aún un tema importante de las actividades propias de la vida rural (Carod 2011) y es quizá por ello que México sea uno de los países latinoamericanos que más ha investigado sobre este grupo de organismos.

\section{Estudios sobre hongos en Centroamérica}

Los países de la región centroamericana cuya riqueza fúngica ha sido mayormente explorada son Costa Rica (2,000 sp) (Mata 2,003), Panamá (1,807 sp) (Piepenbring 2,007) y Guatemala (355 sp) (Flores et al. 2012). En Nicaragua, los primeros estudios sobre hongos se remontan a 1856 y consta de algunos registros realizados por el botánico Charles Wright, que registró algunas especies principalmente del Pacífico nicaragüense (Delgado 2011).

Posteriormente se han ido incrementando los registros de especies que algunos pocos autores han publicado o han ido dejando registros herbarizados y/o dejado investigaciones en algunas bibliotecas del país tales como UNAN-Managua, Herbario Nacional UCA y archivos del centro de Biodiversidad de MARENA.

En Nicaragua, de forma general se reportan 275 especies de Líquenes (Breuss 2002), 194 Hyphomycethes (Delgado 2011) y 184 especies de Macrohongos (MARENA, 2015). Estas cifras han ido aumentando en los últimos años con la labor de diferentes taxónomos, pero los registros aún no han sido unificados en una sola base de datos.

\section{Inventario de hongos en el Caribe nicaragüense}

El Caribe nicaragüense es una de las zonas más biodiversas del país (Cayuela y Granzow 2012), ya que éste representa un mosaico de ecosistemas que alberga una importante variedad de biodiversidad en sus selvas bajas, manglares, zonas estuarinas, etcétera. El Caribe
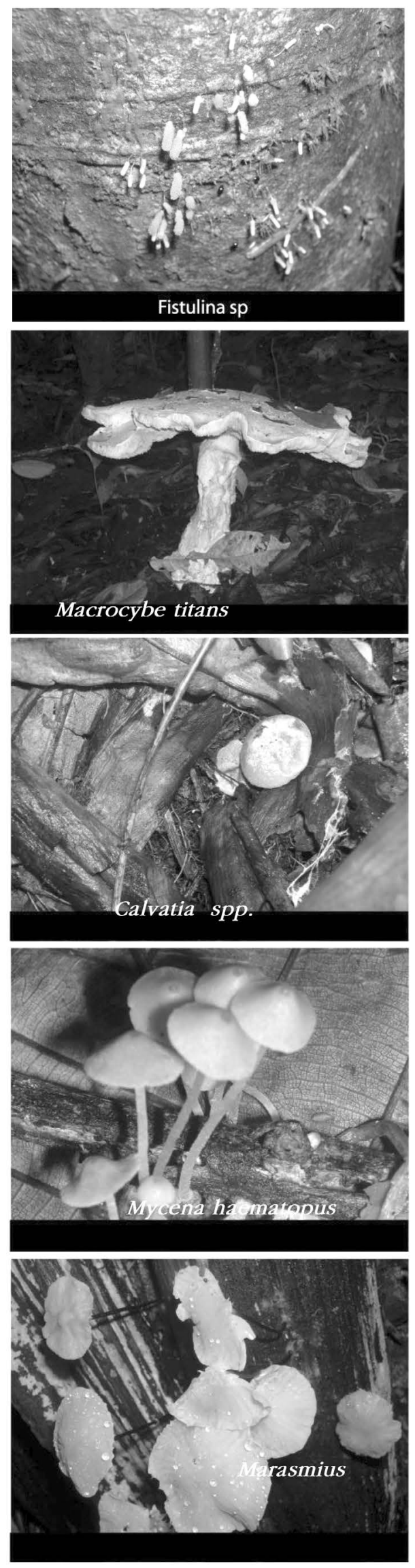
también posee sus particularidades en lo que respecta a la composición de la riqueza fúngica, ya que, por ejemplo, muchas de las especies que en esta zona biogeográfica ocurren son principalmente cosmopolitas o pantropicales (Guzmán 1986), esto influido por los suelos salitrosos y pobres de algunas áreas (Peppenbring y Ruiz 2007).

En Nicaragua, en la última década se han realizado dos estudios importantes sobre hongos en el Caribe nicaragüense, ambos estudios están aislados en el tiempo, sin embargo proveen de una importante base científica que ayuda a entender la composición de la biota fúngica de nuestro Caribe. En el año 2006 concluyó el Inventario de Macrohongos presentes en la Reserva de Biosfera Indio Maíz, Departamento de Río San Juan- Nicaragua (González \& Orozco 2006). Ver anexo 1. Y en el año 2016 se realizó una evaluación de la riqueza y composición de macrohongos en dos biotopos de la estación biológica La Lupe, Reserva Natural Indio Maíz-zona de Amortiguamiento. Ver anexo 2.

El inventario de especies de la Reserva Biológica Indio Maíz del año 2006, corresponde a un trabajo muestreal de treinta días en tres sitios de muestreo con variaciones en la composición florística del biotopo. Los sitios fueron Bartola, Rio Indio y San Juan de Nicaragua. La investigación tuvo una colecta de 1,222 individuos que correspondían a 131 especies de hongos clasificadas en 11 órdenes, 22 familias.

El inventario de hongos que se llevó a cabo en la estación biológica La Lupe consistió en el muestreo de 10 parcelas permanentes de $100 \mathrm{~m}^{2}(10 \mathrm{~m} \times 10 \mathrm{~m})$, equivalentes a una hectárea de terreno en estudio. Las parcelas fueron ubicadas en biotopos diferentes (pastizal y bosque de galería). Se colectaron 536 individuos, representados en 38 especies.

Este documento es un resumen de ambas investigaciones en donde se presenta una lista de las especies de hongos encontradas en ambos estudios y se muestran con especial interés algunas especies que tienen importancia por su comestibilidad, tales como: Auricularia auricula, Auricularia mesentérica, Clavariadelphus truncatus, Coprinus disseminates, Hexagonia hydnoides, Hygrocybe miniata, Hydnum repandum, Psilocybe cubensis y Psilocybe mexicana, estas dos últimas especies son poderosos alucinógenos. En las fotos del Anexo 3., se pueden encontrar varias de las especies aquí mencionadas.
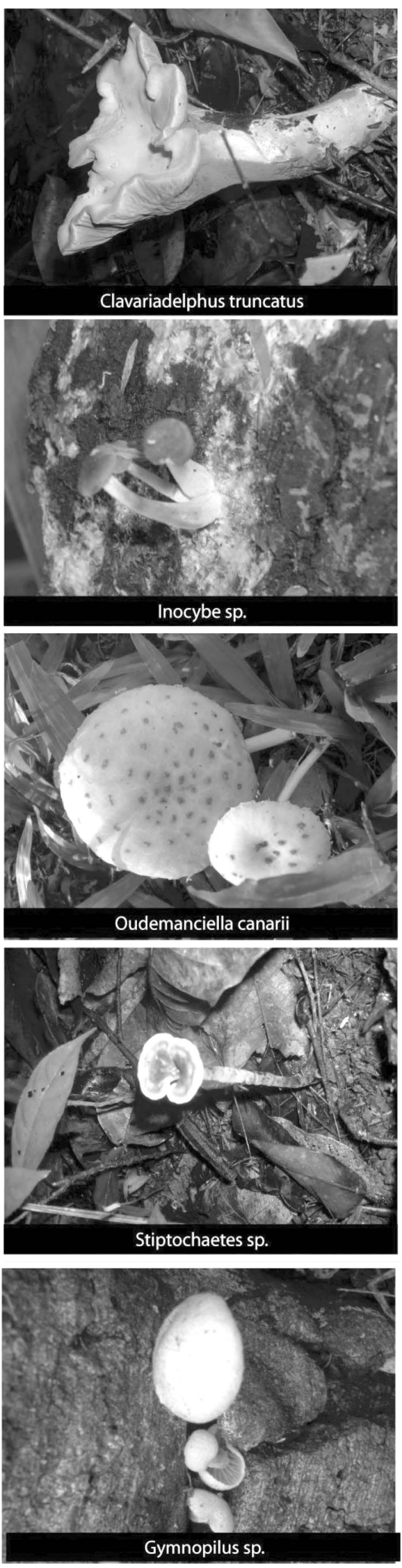


\section{Bibliografía}

Breuss O (2002) Flechten aus Nicaragua. Disponible en

:http://www.landesmuseum.at/pdf_frei_remote/LBB_0034_2_1053-1069.pdf.

Bolaños AC, Soto EM (2011). "Macrohongos comestibles y medicinales comunes en la Universidad del Va1le". Revista de Ciencias Universidad del Valle 86: 45-54.

Cayuela, L. \& Granzow C. (2012). "Biodiversidad y conservación de los bosques neotropicales". Ecosistemas $21: 1-2$

Carod, A. (2015). "Hallucinogenic drugs in pre-Columbian Mesoamerican cultures". Neurobiologia. 30 (1):4249.

Delgado, G. (2011). Nicaraguan fungi; a checklist of hyphomycetes. Disponible en:http://www.mycotaxon. com/resources/checklists/delgado-v115-checklist.pdf.

Egli, S. (2011). "Mycorrhizal mushroom diversity and productivity - An indicator -". Anals for forest Sciense 68: $81-88$.

González, I. \& Orozco, T. (2007). Inventario de Macrohongos Reserva Natural Indio Maíz. Documento inédito. Managua: Araucaria Río San Juan-Ministerio de Ambiente y Recursos Naturales.

Guzmán, G. (1986). "Distribución de los hongos en la región del Caribe y zonas vecinas“. Caldasia 15: 71-75.

Instituto de Biodiversidad -INBIO Costa Rica (2009) Protocolo Hongos: Disponible en: http://www.inbio.ac.cr/ web herbarios/web/pdf/protocolo-hongos.pdf.

Flores AR, Comandini O, Rinaldi AC. (2012). "A preliminary checklist of macrofungi of Guatemala, with notes on edibility and traditional knowledge". Mycosphere 3:1-21.

Hawksworth DL, Rossman AY (1997). “Where are all the undescribed Fungi?”. Phytopatology 87: 888-891.

Ministerio de Recursos Naturales y del Ambiente MARENA, Nicaragua (2014). Biodiversidad en Nicaragua. Disponible en: http://www.bio-nica.info/biblioteca/MARENABiodiversidadNicaragua.pdf

Mata, M. (2003). Macrohongos de Costa Rica; Costa Rica mushrooms, Vol.1, 2da Edition-Instituto de Biodiversidad (INBIO), Santo Domingo de Heredia, Costa Rica.

Pegler, DN (1983) Agaric flora of the Lesser Antilles-Kew Bulletin, Additionals Series IX. Her majestys Stationary Office, London.

Piepenbring M (2007) Inventory the Fungi of Panama-Biodiversity and Conservation 16: 73-84.

Piepenbring M, Ruiz BA (2008) Diversity and ecology of fungi in the Golfo Dulce region Diversidad y ecología de hongos en la región del Golfo Dulce-Stapfia 80: 179-192.

Piepenbring M (2012) Fadenwesen in vielfältigen Nahrungsnischen-Tropische Pilze-Biologie in unserer Zeit 42: 294-301. 


\section{Anexos}

Tabla 1: Listado de especies presentes en la Reserva Biológica Indio Maíz, 2006. González, I. \& Orozco T.

\section{Macrohongos de la Reserva Biologiea Indio Maiz}

\begin{tabular}{|c|c|c|c|}
\hline & Usos & Sustrato & Agrupación \\
\hline $\begin{array}{l}\text { Amauroderma calcigenum (Berk.) Torrend } \\
1920\end{array}$ & Desconocido & Madera & solitario \\
\hline Amauroderma spp. & Desconocido & Madera & Solitario/esparcido \\
\hline Auricularia mesenterica (Pers.) & Comestible & Tierra & Gregario \\
\hline Auricularia auricula-judae (Bull. : Fr.) Quél. & Comestible & Madera & Gregario \\
\hline Auricularia fuscosuccinea Mont. Henn & Comestible & Madera & Gregario \\
\hline Aurificaria luteoumbrina (Romell) D.A. & Desconocido & Madera & Esparcido \\
\hline Aurificaria spp. & Desconocido & Madera/humus & Esparcido \\
\hline Bjerkandera adusta (Will.:Fr) P.Karst & Plaga & Madera & Solitari \\
\hline Caripia montagnei (Berk.) Kuntze & Silvicultura & Madera & Gregario \\
\hline Ceriporia spp. & Desconocido & Tierra & Esparcido \\
\hline Clavaria vermicularis Scop. & Desconocido & Tierra & Esparcido/solitario \\
\hline Clavaria spp. & Desconocido & Madera & Esparcido \\
\hline Clavariadelphus truncatus (Quél.) Donk & comestible & Madera tierra & Esparcido \\
\hline Clavaria laeticolor Berk. \& M.A.Curtis & Desconocido & Madera & Gregario \\
\hline $\begin{array}{l}\text { Chlorophyllum molybdites (G. Meyer : Fr.) } \\
\text { Massee }\end{array}$ & Desconocido & Tierra & Esparcido \\
\hline Clavicorona spp. & Desconocido & Madera & Gregario \\
\hline Collybia aurea (Beeli) Pegler & Desconocido & Madera & Gregario \\
\hline Collybia dryophila. (Bull) P. Kumm., Fuehr. & Desconocido & Tierra & Esparcido \\
\hline Collybia neotropica Singer, Sydowia & Desconocido & Tierra & Madera/ Gregario \\
\hline Coltricia hamata (Romell) Ryvarden & Desconocido & Madera & Esparcido \\
\hline Coltricia spp. (1) & Desconocido & Madera & Solitario \\
\hline Coltricia spp. (2) & Desconocido & Humus & Esparcido \\
\hline Cookeina speciosa Kuntze & Investigación & Madera & Gregario \\
\hline Cookeina tricholoma (Mont.) Kuntze & Investigación & Madera/Tierra & Esparcido \\
\hline Coprinus disseminatus (Pers.) Gray & Comestible & Madera/Tierra & Gregario \\
\hline Coprinus spp. & Desconocido & Tierra & Esparcido \\
\hline Cyathus striatus (Huds) & Desconocido & Madera & Gregario/esparcido \\
\hline Daedalea quercina (L.) Pers. & Industria & Madera & Gregario \\
\hline
\end{tabular}




\begin{tabular}{|c|c|c|c|}
\hline Datronia caperata (Berk.) Ryvarden & Desconocido & Madera & Gregario \\
\hline $\begin{array}{l}\text { Dacryopinax spathularia (Schwein.) G.W. } \\
\text { Martin }\end{array}$ & Desconocido & Madera & Gregario \\
\hline Dictyopanus pusillus (Pers. ex Lév.) Singer & Desconocido & Madera & Gregario \\
\hline Earliella scabrosa (Pers.) Gilb. \& Ryvarden & Desconocido & Madera & Tierra \\
\hline Filoboletus gracilis (Klotzsch ex Berk.) Singer & Desconocido & Madera & Esparcido \\
\hline Ganoderma lucidum (Curtis) P. Karst & Medicinal & Madera & Esparcido \\
\hline Ganoderma applanatum (Pers.) Pat. & Desconocido & Madera & Solitario \\
\hline Ganoderma australe (Fr.) Pat & Desconocido & Tierra & Gregario \\
\hline Ganoderma spp. & Desconocido & Tierra & Solitario \\
\hline Gloeophyllums spp. & Desconocido & Madera & Esparcido \\
\hline Gymnopilus spp. & Desconocido & Madera & Esparcido \\
\hline Grifola spp. & Desconocido & Madera & Solitario \\
\hline Heterobasidion annosum (Fr.) Bref. & Investigación & Madera & Esparcido \\
\hline Hexagonia hydnoides (Sw.) M. Fidalgo & Medicinal & Madera & Esparcido \\
\hline Hohenbuehelia spp. & Desconocido & Tierra & Esparcido \\
\hline Hygrocybe miniata (P.D.Orton) Svrcek & Comestible & Tierra & Gregario \\
\hline Hygrocybe colemaniana Brun & Desconocido & Tierra & Solitario \\
\hline Hydnum repandum L:Fr. & Comestible & Tierra & Esparcido \\
\hline Humphreya coffeatum (Berk.) Steyaert & Desconocido & Tierra & Solitario \\
\hline Inocybe calamistrata (Fr.) Gillet & Venenoso & Madera & Esparcido \\
\hline Inocybe spp. & Desconocido & Madera & Esparcido \\
\hline Inonotus dryadeus Pers.:Fr & Desconocido & Tierra & Solitario \\
\hline Irpex spp.(1) & Desconocido & Madera & Esparcido \\
\hline Irpex spp. (2) & Desconocido & Tierra & Gregario \\
\hline Laccaria ohiensis (Mont) Singer. & Desconocido & Madera & Solitario \\
\hline Laccaria spp. & Desconocido & Madera & Esparcido \\
\hline Laetiporus spp. & Desconocido & Madera & Solitario \\
\hline Lentinus tigrinus (Bull.) Fr. & Desconocido & Madera & Esparcido \\
\hline $\begin{array}{l}\text { Lentinula aciculospora J.L.Mata \& R.H.Peter- } \\
\text { sen }\end{array}$ & Desconocido & Tierra /Madera & Esparcido \\
\hline Lepiota subcristata Cleland & Comestible & Madera & Tierra \\
\hline Lepiota ianthinoscuamosa singer. & Venenosa & Madera & Solitario/esparcido \\
\hline Lepista flaccida (Sowerby) Pat. & Venenosa & Tierra & Solitario/esparcido \\
\hline Lycoperdon perlatum, syn. L. gemmatum & Comestible & Tierra & Solitario \\
\hline
\end{tabular}




\begin{tabular}{|c|c|c|c|}
\hline Lycoperdon piriforme Schaeff .:Pers. & Comestible & Tierra & Esparcido \\
\hline $\begin{array}{l}\text { Leucocoprinus fragilissimus (Berk. \& M.A. } \\
\text { Curtis) Pat. }\end{array}$ & Desconocido & Tierra & Solitario \\
\hline Leucocoprinus sulphureus (Schnizl.) Cejp & Desconocido & Madera y Tierra & Gregario y Esparcido \\
\hline Marasmius aripoensis (Dennis) Singer. & Desconocido & Tierra & Gregario \\
\hline Marasmius cladophyllus (Berk). & Desconocido & Tierra & Esparcido \\
\hline Marasmius haematocephalus (Mont.) Fr. & Desconocido & Tierra & Gregario \\
\hline Marasmius guadalupensis Pat. & Desconocido & Madera & Gregario \\
\hline Marasmius perlongispermus Singer & Desconocido & Tierra & Esparcido \\
\hline Marasmius johnstonii Murrill & Desconocido & Madera & Esparcido \\
\hline Marasmius hakgalensis var. Hakgalensis & Desconocido & Madera & Esparcido \\
\hline Marasmius spp & Desconocido & Madera & Esparcido \\
\hline $\begin{array}{l}\text { Marasmiellus tricolor (Alb. \& Schwein.) } \\
\text { Singer }\end{array}$ & Desconocido & Tierra & Esparcido \\
\hline Marasmius volvatus & Desconocido & Tierra & Gregario \\
\hline Mycena haematopus (Pers. : Fr.) P. Kumm. & Desconocido & Tierra & Esparcido \\
\hline Mycena margarita (Murrill) Murrill & Desconocido & Tierra & Esparcido \\
\hline $\begin{array}{l}\text { Mycena sanguinolenta (Alb. \& Schwein. : Fr.) } \\
\text { P. Kumm }\end{array}$ & Venenosa & Tierra & Gregario \\
\hline Mycena spp. & Desconocido & Madera & \\
\hline Meripilus spp. & Desconocido & Tierra & Solitario/esparcido \\
\hline Megacollybia spp. & Desconocido & & \\
\hline Onnia tomentosa (Fr.) P.Karst. & Desconocido & Madera & Esparcido/solitario \\
\hline Oudemansiella canarii (Jungh.) Höhn. & Desconocido & & \\
\hline Oxyporus spp. & Desconocido & Madera & Esparcido \\
\hline Panaeolus antillarum (Fr.) Dennis & Venenoso & Tierra & Esparcido/solitario \\
\hline Panaeolina foenisecii (Pers. : Fr.) Maire & Tóxico & Tierra & Solitario \\
\hline Panus crinitus (L. ; Fr.) Fr. & Desconocido & Madera & Esparcido \\
\hline Phallus indusiatus Vent. & Comestible & Madera & Esparcido \\
\hline $\begin{array}{l}\text { Phellinus longisetulosus Bondartseva \& S. } \\
\text { Herrera }\end{array}$ & Desconocido & Madera & Esparcido \\
\hline Phylicia cuculiformes montagne & Desconocido & Madera & Gregario \\
\hline Phylotus porrigens (Pers.:Fr.) & Tóxico & Madera & Esparcido \\
\hline Polyporus arcularius (Batsch) Fr. & Investigación & Madera & Esparcido \\
\hline Polyporus badius (Pers.) Schwein. & Desconocido & Tierra & Gregario \\
\hline Polyporus elegans (Bull.) Trog & Desconocido & Madera & Esparcidos \\
\hline
\end{tabular}




\begin{tabular}{|c|c|c|c|}
\hline $\begin{array}{l}\text { Polyporus occidentalis (Murrill) Sacc. \& Trot- } \\
\text { ter }\end{array}$ & Desconocido & Madera & Esparcido \\
\hline Polyporus tenuiculus (P. Beauv.) Fr. & Investigación & Madera & Esparcido \\
\hline Polyporus tricholoma Mont. & Comestible & Madera & Esparcido a Gregario \\
\hline Polyporus mori (Pollini) Fr. & Desconocido & Madera & Esparcido \\
\hline Polyporus leprieurii Mont. & Silvicultura & Madera & Gregario \\
\hline Polyporus spp.(1) & Desconocido & Tierra & Solitario \\
\hline Polyporus spp.(2) & Alucinogeno & Tierra & Gregario \\
\hline Psilocybe mexicana R.Heim & Alucinogeno & Tierra & Gregario \\
\hline Psilocybe cubensis (Earle) Singer & Desconocido & Tierra & Solitario \\
\hline Psilocybe coprophila (Bull.) P. Kumm. & Desconocido & Tierra & Gregario \\
\hline Pycnoporus sanguineus (L.) Murrill & Medicinal & Madera & Gregario \\
\hline Perenniporia spp. & Desconocido & Tierra & Esparcido \\
\hline Physisporinus vitreus (Pers.) P.Karst. & Desconocido & Madera & Esparcido \\
\hline Paxillus spp. & Tóxico & Madera & Gregario \\
\hline Perenniporia spp. & Desconocido & Tierra & Gregario \\
\hline Phaeolus spadiceus (Pers. : Fr.) Rauschert & Desconocido & Tierra & Esparcido \\
\hline Physisporinus spp. & Desconocido & Madera & Esparcido \\
\hline Ripartiella alba & Desconocido & Madera & Gregario \\
\hline Rigidoporus spp.(1) & Desconocido & Madera & Esparcido \\
\hline Rigidoporus spp.(2) & Desconocido & Madera & Esparcido \\
\hline Strobilurus conigenoides(Ellis) Singer & Desconocido & Tierra & Gregario \\
\hline Schizophyllum commune Fries & Desconocido & Madera & Gregario \\
\hline Trametes versicolor (L.:Fr.) Quél. & Investigación & Madera & Esparcido \\
\hline Tetrapyrgos nigripis E.Horak & Investigación & Madera & Esparcido \\
\hline Tricholomopsis spp.(1) & Desconocido & Madera & Gregario \\
\hline Tricholomopsis spp. (2) & Desconocido & Madera & Gregario \\
\hline Wolfiporia spp. & Desconocido & Tierra & Esparcido \\
\hline Xeromphalina tenuipes Miller & Desconocido & Madera & Gregario \\
\hline $\begin{array}{l}\text { Xeromphalina campanella (Batsch) Kühner \& } \\
\text { Maire }\end{array}$ & Desconocido & Madera & Esparcido \\
\hline Xylaria hypoxylon (L.) Grev. & Desconocido & Madera & Gregario \\
\hline Xylaria polymorpha (Pers.) Grev & Desconocido & Madera & Gregario \\
\hline Xylaria spp. (1) & Desconocido & Madera & Gregario \\
\hline Xylaria spp (2) & Desconocido & Madera & Gregario \\
\hline
\end{tabular}


Tabla 2. Listado de especies presentes en diferentes Biotopos de la Estación Biológica la Lupe, Rio San Juan-Nicaragua Diciembre 2016. Saldivar, I.

\begin{tabular}{|c|c|c|c|c|c|c|}
\hline \multicolumn{7}{|c|}{ Listado de Macrohongos de la Estacion Biologica la Lupe, Oetuhre 2016} \\
\hline & Especies & Pastizal & Bosque & Terrestre & Madera sap & Hoja \\
\hline 1 & Auricularia auricula-judae (Bull. : Fr.) Quél. & & $\mathrm{B}$ & & $\mathrm{M}$ & \\
\hline 2 & Caripia montagnei (Berk.) Kuntze & & $\mathrm{B}$ & & $\mathrm{M}$ & \\
\hline 3 & Cyptotrama asprata (Berk.) Redhead \& Ginns & $\mathrm{P}$ & $\mathrm{B}$ & & $\mathrm{M}$ & \\
\hline 4 & Calvatia sp. & $\mathrm{P}$ & & & $\mathrm{M}$ & \\
\hline 5 & Clitocybe sp. & & $\mathrm{B}$ & $\mathrm{T}$ & & \\
\hline 6 & Collybia aurea (Beeli) Pegler & & $\mathrm{B}$ & & $\mathrm{M}$ & \\
\hline 7 & Collybia sp & $\mathrm{P}$ & $\mathrm{B}$ & & $\mathrm{M}$ & \\
\hline 8 & Cookeina tricholoma Kuntze & $\mathrm{P}$ & $\mathrm{B}$ & & $\mathrm{M}$ & \\
\hline 9 & Cookeina speciosa (Fr.) Dennis & & $\mathrm{B}$ & & $\mathrm{M}$ & \\
\hline 10 & Coprinellus disseminatus (Pers.) J.E.Lange & & $\mathrm{B}$ & & $\mathrm{M}$ & \\
\hline 11 & Coprinus plicatilis (Curtis) Fr. & & $\mathrm{B}$ & & & $\mathrm{H}$ \\
\hline 12 & Earliella scabrosa (Pers.) Gilb. \& Ryvarden & & $\mathrm{B}$ & & $\mathrm{M}$ & \\
\hline 13 & Dictyopanus pusillus (Pers. ex Lév.) Singer & $\mathrm{P}$ & & & $\mathrm{M}$ & \\
\hline 14 & Ganoderma australe (Fr.) Pat. & $\mathrm{P}$ & & & M & \\
\hline 15 & Hohenbuehelia angustata (Berk.) Singer & B & & M & & \\
\hline 16 & Hymenochaete luteo badia (Fries). & $\mathrm{B}$ & & $\mathrm{M}$ & & \\
\hline 17 & Hymenogloea papyracea (Berk. \& M.A. Curtis) Singer & $\mathrm{P}$ & $\mathrm{B}$ & $\mathrm{T}$ & & \\
\hline 18 & Leucocoprinus cepistipes (Sowerby) Pat. & $\mathrm{P}$ & $\mathrm{B}$ & $\mathrm{T}$ & & \\
\hline 19 & Leucocoprinus fragilissimus (Berk. \& M.A.Curtis) Pat. & B & & M & & \\
\hline 20 & Lepiota iantinosquamosa Singer. & & $\mathrm{B}$ & $\mathrm{T}$ & & \\
\hline 21 & Lepiota subcristata Cleland & $\mathrm{P}$ & $\mathrm{B}$ & & $\mathrm{M}$ & \\
\hline 22 & Lycoperdon pyriforme Schaeff. : Pers. & & $\mathrm{B}$ & $\mathrm{T}$ & & \\
\hline 23 & Marasmius aripoensis Pegler. & & $\mathrm{B}$ & & $\mathrm{M}$ & \\
\hline 24 & Marasmius berteroi (Lév.) Murrill & & $\mathrm{B}$ & & $\mathrm{M}$ & \\
\hline 25 & Marasmius cladophyllus Berk & $\mathrm{P}$ & $\mathrm{B}$ & & $\mathrm{M}$ & \\
\hline 26 & Marasmius perlongispermus Singer & $\mathrm{B}$ & & $\mathrm{M}$ & & \\
\hline 27 & Megacollybia platyphylla (Pers. : Fr.) Kotl. \& Pouzar & $\mathrm{B}$ & $\mathrm{T}$ & & & \\
\hline 28 & Mycena pallida (Murrill) A.H. Sm. & & $\mathrm{B}$ & & $\mathrm{M}$ & \\
\hline 29 & Psathyrella conopilus Batsch. & $\mathrm{P}$ & $\mathrm{B}$ & & $\mathrm{M}$ & \\
\hline 30 & Pluteus sp & $\mathrm{P}$ & $\mathrm{B}$ & & $\mathrm{M}$ & \\
\hline 31 & Polyporus tenuiculus (P. Beauv.) Fr. & & $\mathrm{B}$ & & $\mathrm{M}$ & \\
\hline 32 & Ramaria stricta (Pers.) Quél. & & $\mathrm{B}$ & $\mathrm{T}$ & & \\
\hline 33 & Resupinatus sp & & $\mathrm{B}$ & & $\mathrm{M}$ & \\
\hline 34 & Schizophyllum commune Fr. & $\mathrm{P}$ & $\mathrm{B}$ & & $\mathrm{M}$ & \\
\hline 35 & Trametes versicolor (L.) Lloyd & $\mathrm{P}$ & $\mathrm{B}$ & & $\mathrm{M}$ & \\
\hline 36 & Xylaria hypoxylon (L.) Grev. & & $\mathrm{B}$ & & $\mathrm{M}$ & \\
\hline 37 & Xylaria polymorpha (Pers.) Grev & & $\mathrm{B}$ & & $\mathrm{M}$ & \\
\hline 38 & Xylaria sp. & & $\mathrm{B}$ & & $\mathrm{M}$ & \\
\hline
\end{tabular}


Fotos.3. Macrohongos Reserva Biológica -Indio Maiz 2006
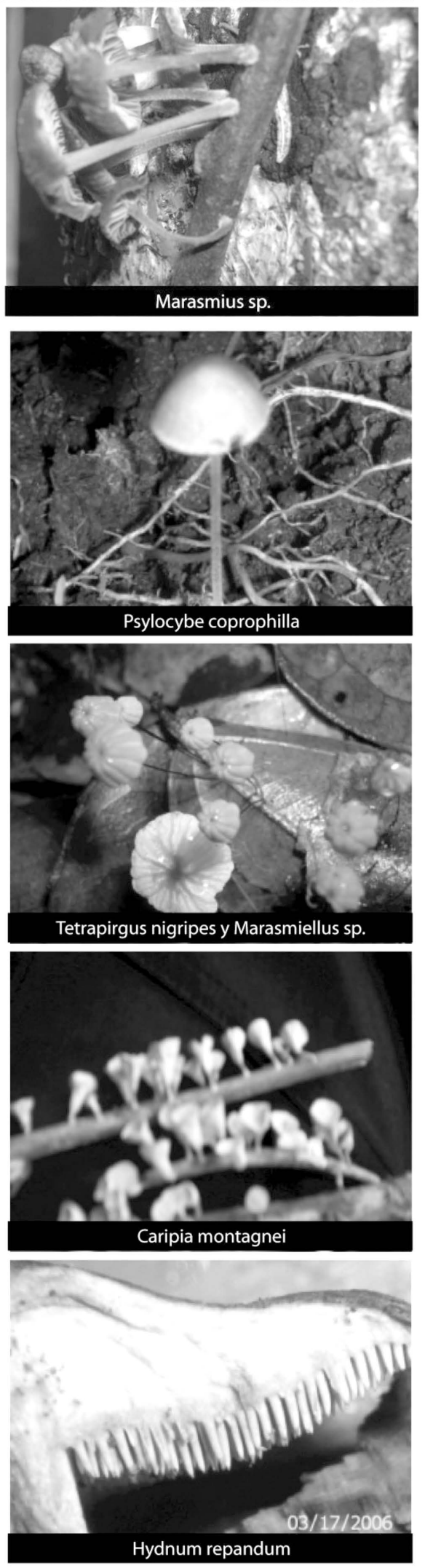
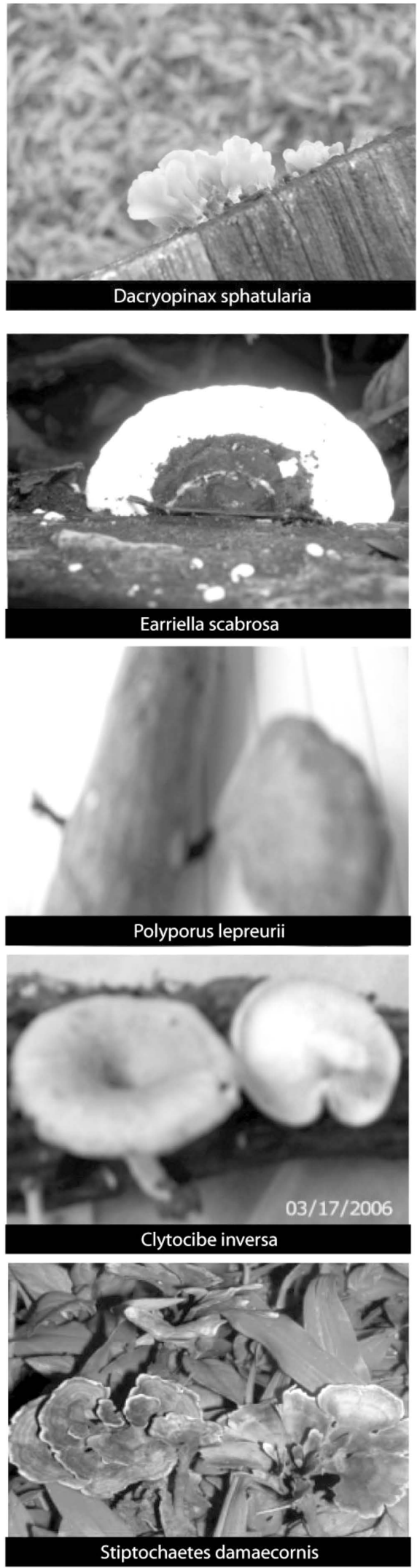
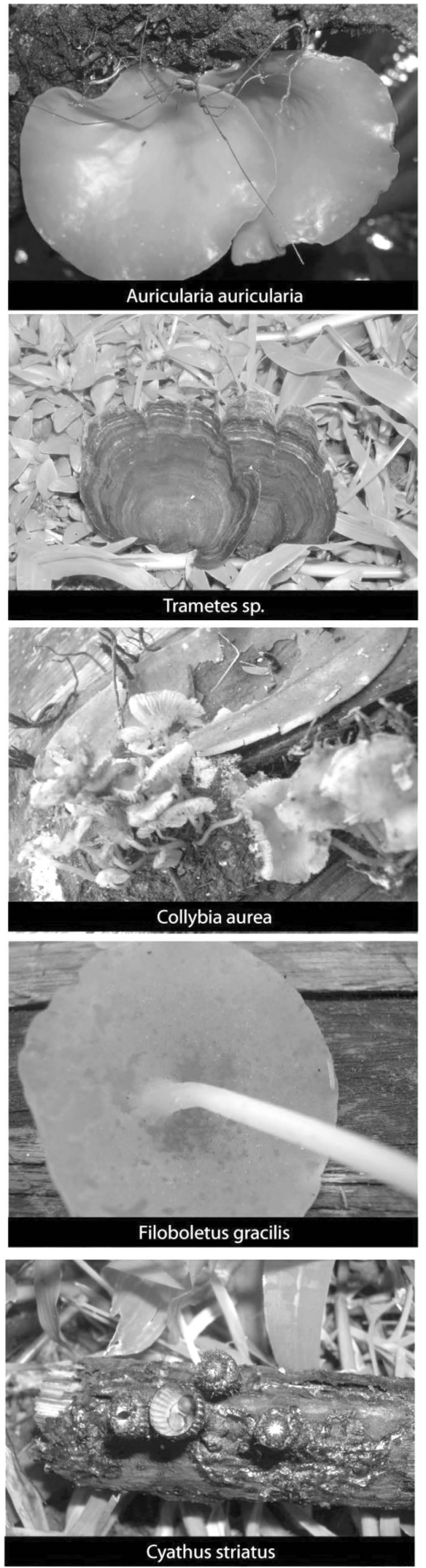


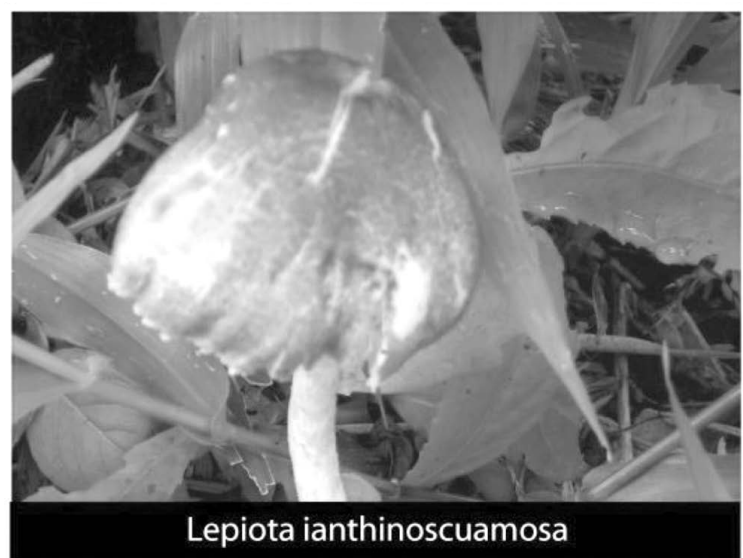

Xylaria Clata

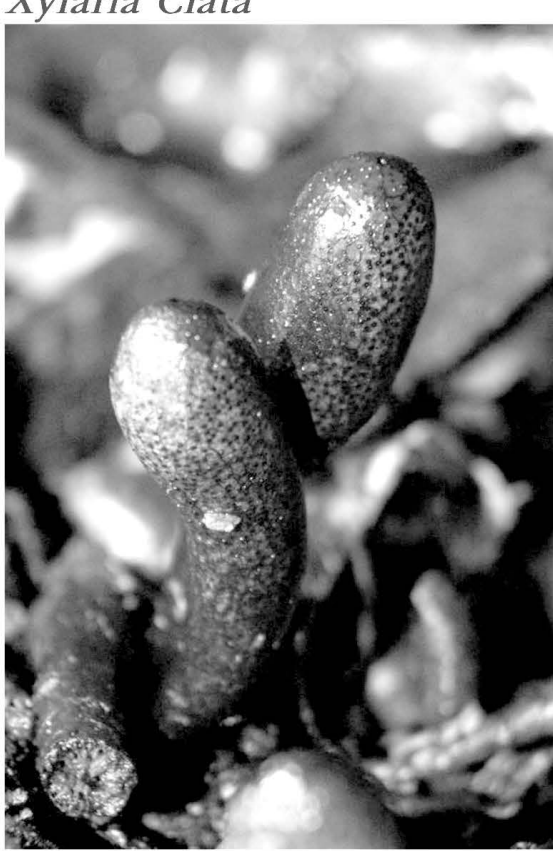

Inocybe calamistrata

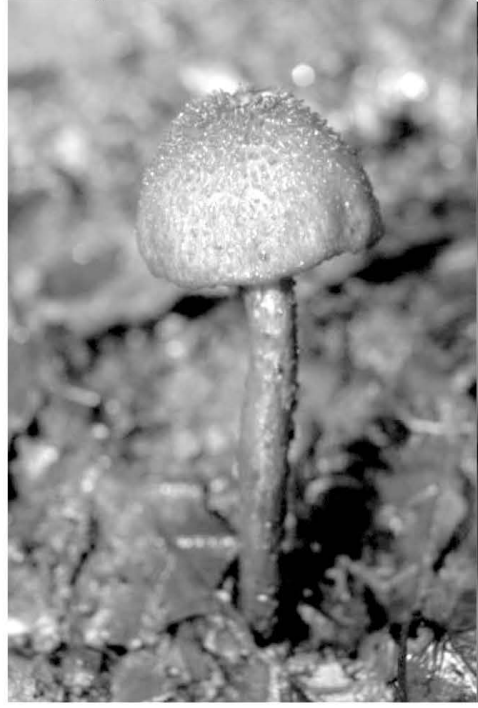

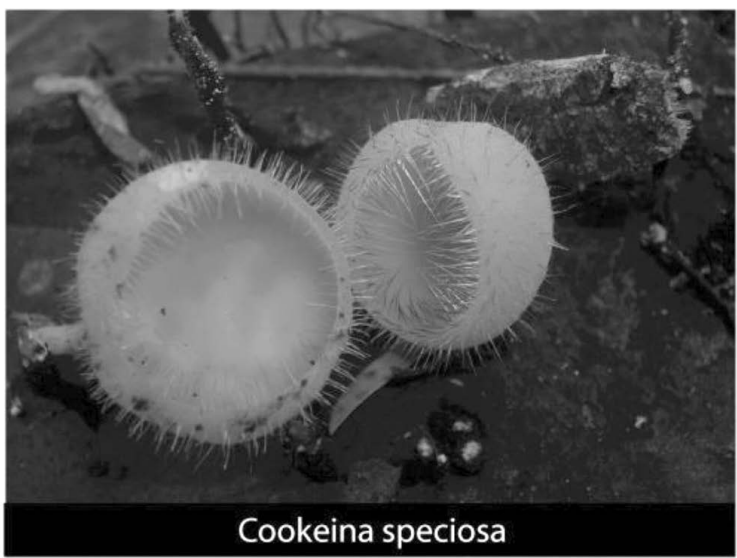

Marasmius berteroi

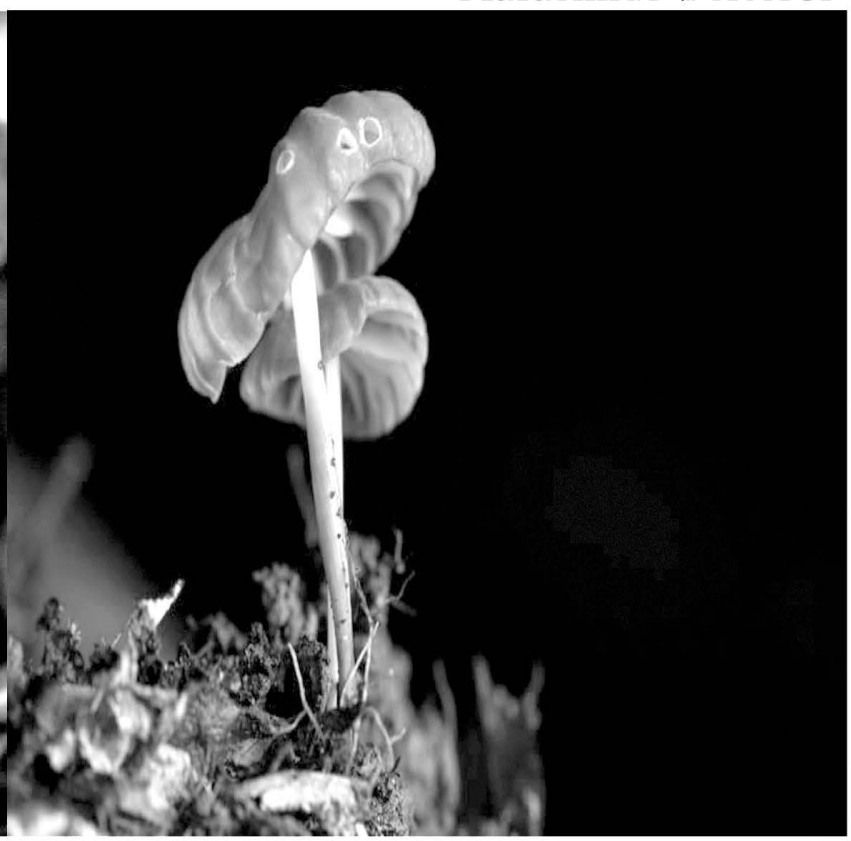

Pasatyrella conopillus

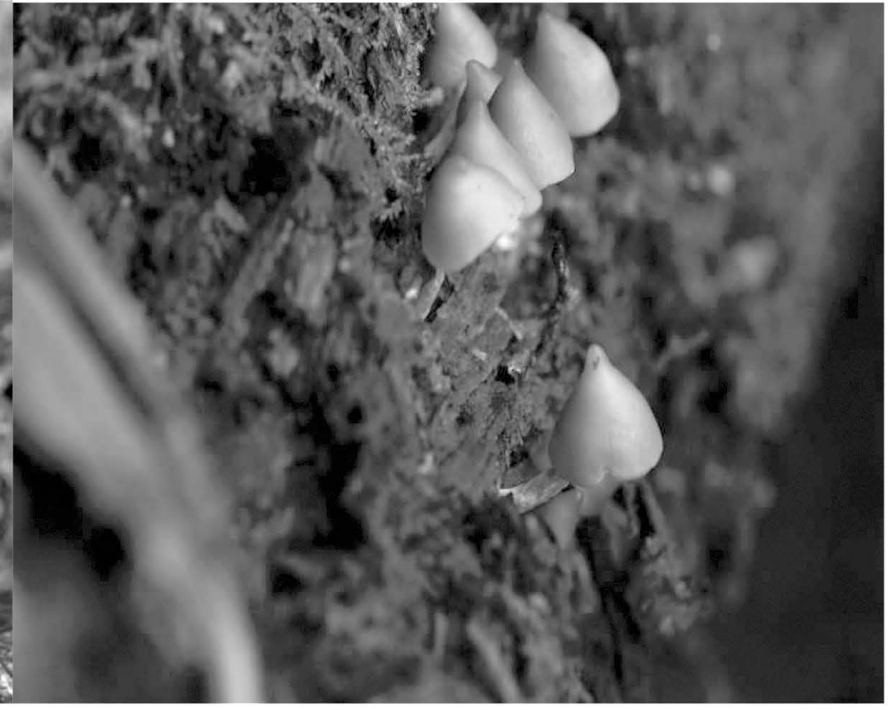

